\title{
Bering Mission Navigation Method
}

Betto, Maurizio; Jørgensen, John Leif; Jørgensen, Peter Siegbjørn; Denver, Troelz

Published in:

Proceedings of the RAST Conference

Link to article, DOI:

10.1109/RAST.2003.1303917

Publication date:

2003

Document Version

Publisher's PDF, also known as Version of record

Link back to DTU Orbit

Citation (APA):

Betto, M., Jørgensen, J. L., Jørgensen, P. S., \& Denver, T. (2003). Bering Mission Navigation Method. In Proceedings of the RAST Conference (pp. 239-244). IEEE. https://doi.org/10.1109/RAST.2003.1303917

\section{General rights}

Copyright and moral rights for the publications made accessible in the public portal are retained by the authors and/or other copyright owners and it is a condition of accessing publications that users recognise and abide by the legal requirements associated with these rights.

- Users may download and print one copy of any publication from the public portal for the purpose of private study or research.

- You may not further distribute the material or use it for any profit-making activity or commercial gain

- You may freely distribute the URL identifying the publication in the public portal

If you believe that this document breaches copyright please contact us providing details, and we will remove access to the work immediately and investigate your claim. 


\title{
BERING MISSION NAVIGATION METHOD
}

\author{
M. Betto, J.L. Jørgensen, P.S. Jergensen, and T. Denver \\ Ørsted-DTU, MIS, Building 327, Technical University of Denmark, DK-2800 Kgs. Lyngby, \\ Phone +45 4525 3598, Fax +45 45887133 , \\ email mbe@oersted.dtu.dk, jlj@oersted.dtu.dk,psj@oersted.dtu.dk, td@oersted.dtu.dk
}

\begin{abstract}
"Bering", after the name of the famous Danish explorer, is a Near Earth Object (NEO) and main belt asteroids mapping mission envisaged by a consortium of Danish universities and research institutes. To achieve the ambitious goals set forth by this mission, while containing the costs and risks, "Bering" sports several new technological enhancements and advanced instruments under development at the Technical University of Denmark (DTU). The autonomous on-board orbit determination method is part of them and it is described in this paper.
\end{abstract}

-.- Key words: Navigation, orbit determination, advanced stellar compass.

\section{INTRODUCTION}

The scientific rationale of "Bering" is described in (1), (2) \& (10). The mission profile and conceptual spacecraft configuration and operations are presented in (5), (11) \& (14). In short, "Bering" is a deep space mission to map the populations of the NEO and of the main belt asteroids and to derive information about the associated transportation mechanism.

Composed by a fleet of two spacecraft, "Bering" will initially fly inward the solar system to map the NEO population and to fly-by Venus to obtain the energy to fly out to the main asteroid belt The conceptual mission profile is sketched in fig.l.

Navigation is the Achilles' heel of deep space. Being performed on ground, it imposes considerable constrains on the system and the operations, it is very expensive to execute, especially when the mission lasts several years and, above all, it is not failure tolerant. These drawbacks are aggravated when more than one spacecraft are to be controlled. Therefore, in order to reduce the costs and complexity of the mission while increasing the flexibility, science return and robustness, the autonomous onboard orbit determination method developed at DTU is baselined for "Bering".

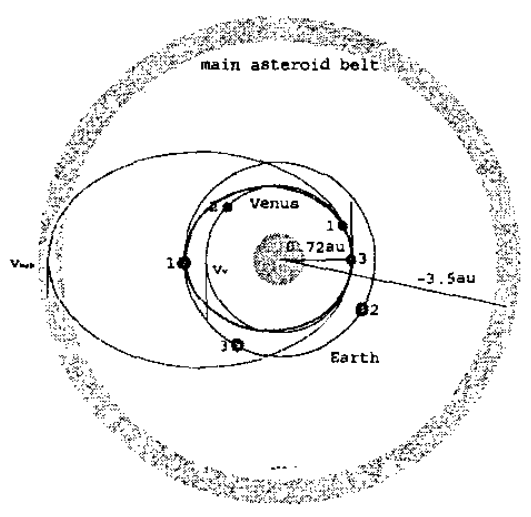

Figure 1. The "Bering" mission profile

This method, devised by the authors, does not require any a-priori knowledge of any kind. The solution is robust, elegant and fast and it is possible because of the technological breakthrough of advanced star trackers, like the micro-Advanced Stellar Compass ( $\mu \mathrm{ASC}$ ) into which it is implemented.

\section{WHAT IS NAVIGATION}

Space navigation is the process by which the position and the velocity, over time, of a spacecraft are estimated with respect to a reference frame at any epoch. Up to date, the space navigation is performed from ground. The measurements, taken by the tracking stations, are processed and propagated with the results of the integration of the equations of motion, that take the known forces acting on the system into account, to forecast the spacecraft state. Then, the predicted and the nominal trajectories are compared to check whether corrective actions are called for. This is usually the case because errors of various origins influence the prediction of the state as well as the definition of the nominal profile. A new course is then evaluated, the orbit corrections are planned and transmitted to the spacecraft to be, normally, executed in open loop. Hence, the measurements and estimations need to be very 
accurate.

The most common method of ground based deep space navigation rests on radar tracking. Ground tracking stations normally use all the available data and orbit models. Hundreds or thousands of measurements are always available and filtering techniques, like the least square method or Kalman filters, are used to process them and to improve the accuracy. These methods manipulate a wealth of measurements of different nature: a) range measurements; b) Doppler shift; c) angular measurements. The latter are seldom used for deep space because they degrade with scale.

Ground tracking drives the mission costs and are not robust towards loss of contact, space segment degradations etc.. The costs are both direct and indirect. Direct costs are those related to the use and operations of the ground stations for extended periods of time. Indirect costs are those associated with the increased complexity of the spacecraft due to the constraints imposed at system level to guarantee that the vehicle can always be tracked from ground and to reduce the risk of loss of contact. The robustness of the mission against mishaps mainly relies on preventing hazardous situations. This is achieved by constraining the system design, the trajectory planning and the attitude maneuvers.

\section{AUTONOMOUS NAVIGATION}

Autonomous navigation is the process by which the spacecraft's orbital parameters are estimated on-board the vehicle without relying on aid and guidance, i.e. intervention, from the ground segment. Hence, autonomous navigation cuts the need for a constant communication link to earth. It permits to relax system and operation requirements and to build more flexible and agile spacecrafts. The costs can be considerably reduced and the robustness augmented. In principle, ground and space based navigation could use the same techniques and instruments. However, in practice, this is not possible because the resources, especially power, mass and volume, available on-board a spacecraft are limited. Thus, the equipment used on ground cannot be installed on-board. Furthermore, particularly in case of contingency, much less resources are available to the system to recover the knowledge of its status. Therefore, a different approach based on flyable hardware has to be devised.

\section{THE $\mu$ ASC}

Optical systems provide the right tack to design an instrument for deep space autonomous navigation. Indeed, they are intrinsically more accurate and less demanding, in terms of power and mass/size budgets, than the radar systems and are easier to operate and more robust because they passively acquire the signal coming from the observed body rather than actively track it.

The micro-Advanced Stellar Compass ( $\mu \mathrm{ASC}$ ); is a $4^{\text {th }}$ generation star tracker, featuring full autonomy of all ma-

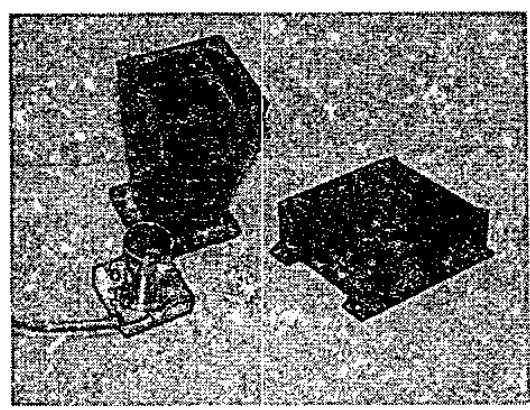

Figure 2. The $\mu A S C$

jor functionalities. The $\mu$ A.SC is described in details in (7), so for this context it is sufficient to repeat a few of the instruments performance characteristics.

The $\mu \mathrm{ASC}$ consists of two separate functional blocks, the Data Processing Unit (DPU) and the Camera Head Unit (CHU), both shown in fig. 2. Despite its miniature size and mass, the $\mu \mathrm{ASC}$ supports two fully redundant powerful DPUs built in. Each of which may drive up to four CHUs. The $\mu \mathrm{ASC}$ uses a cross strapping board to access the CHUs so that the user may configure any of the CHUs to any DPU as the situation may demand. This configuration has been chosen, partly to achieve a useful lifetime of over 30 years in space, partly to allow for more complex processing should the need arise, say if the mission demand very high data availability on special occasions like kick motor.firing or precision manoeuvering, in which case one DPU can be configured with one or two CHUs and the other with the rest.

The use of a multi CHU $\mu$ ASC increases the reliability and relaxes several constraints from the basic spacecraft attitude control, like the avoidance of sun or planet blinding.

\section{1. $\mu \mathrm{ASC}$ Factsheet}

The functionality and budgets of the $\mu \mathrm{ASC}$ are hereby summarized.

- Fully autonomous operations

- Fully resilient to sun illumination

- Radiation tolerant design (>60kRad)

- Separate cameras and processor

- Full cold/hot redundancy

- High reliability (>99.9998\% 3 years redundant configuration)

- In-line imaging capability

- Identification and isolation of non-stellar objects

- Compact Data Processing Unit (DPU) 


$$
\begin{aligned}
& \text { - } 100 \times 100 \times 60 \mathrm{~mm} \\
& \text { - Low mass: } 400 \mathrm{~g} \\
& \text { - Low power: <3.9W @4 CHU \& 22Hz update } \\
& \text { rate }
\end{aligned}
$$

- Up to 22 true solutions per second

- Fast recovery from attitude "lost in space" (LIS) (<30ms, typical $10 \mathrm{~ms}$ )

- "Deep Space Navigation" (DSN) module

- Compact Camera Head Unit (CHU)

$-50 \times 50 \times 50 \mathrm{~mm}$

- Low mass: $280 \mathrm{~g}$

- High tolerance to bright objects ( $>50 \%$ moon in f.o.v.)

- Bias free mounting

- High accuracy

$$
\begin{aligned}
& -\mathrm{NEA}<0.6 \text { "@ } 2 \mathrm{~Hz} \\
& -\mathrm{RA}<1.5 \text { "@ } 2 \mathrm{~Hz} \\
& - \text { Bias }<2 \text { " over the full temperature range }
\end{aligned}
$$

\section{ON THE ORBIT DETERMINATION METHODS}

If the knowledge of the spacecraft position was limited to be able to point the antenna to Earth, then it would be sufficient to periodically upload the spacecraft trajectory in a format that allows a polynomial propagation. However, this approach requires the spacecraft to be in constant or frequent contact with Earth. Hence, it is not robust against long periods of no visibility, is not redundant and it does not materialize the savings related to the cuts of the ground tracking.

To achieve full autonomy, the "lost in space" problem shall be solved on-board anywhere at any time.

The difficulty, in solving the initial orbit determination, arises from the fact that the observations, performed with optical instruments, give only the direction of the object as seen by the observer and do not furnish any direct information about the distance. The position of the body is therefore not given and the velocity is obviously not determined. Hence, it becomes necessary to observe the object again at another time. However, during the time between observations also the observer moves and his motion shall be taken into account to be able to determine the orbit of the object. The solution of this problem presents some difficulties. Kepler, Laplace and Gauss solved the problem. Whether the methods devised by Laplace and Gauss really work and whether they have ever really been used successfully is questioned by some authors (13). Anyway, neither of them is suitable for autonomous navigation because: a) at least three observations of the same object are necessary over a period of time that spans from hours to days; b) the object detected and used to determine the orbit of the spacecraft is not immediately identified, hence any realistic solution shall be maintained until it can be ruled out by other observations; c) more than one non-stellar object can be expected in each image, therefore the number of possible solutions increases rapidly with observations.

A different approach is used by astronomers studying the minor planets of the solar system and searching - among the rest - for objects that may collide with the Earth. This technique sifts asteroids in selected area of the night sky by taking two images of the same field during the same night with a time interval of few hours in between. The images are then scanned to detect moving objects. From two observations, the knowledge of the observer position and some hypotheses about the nature of the object (i.e. the possible distance and velocity), families of solutions are computed and the ephemerides are forecasted. Then, the regions of interest are observed again and if the object is found, one solution is confirmed. This method is clearly unsuitable for autonomous navigation because: a) it is based on some a-priori knowledge; $b$ ) it is not robust; c) it requires a considerable processing time.

\subsection{Deep Space 1}

The first attempt to increase the on-board autonomy by shifting the navigation from ground to the spacecraft was done recently by JPL on-board the Deep Space I (DS-1) mission (see (3) for the details). The principle adopted by DS- 1 for autonomous orbit determination is triangulation. If two asteroids could be observed at once, a triangle could be construed. As the positions of the asteroids are known, the position of the spacecraft can be computed. In practice, this was achieved by pointing the optics to specific targets at pre-defined times. The nominal trajectory - known on-board $\sim$ was compared with the measured position and the corrections computed and applied in order to maintain the pre-defined orbit. The major criticisms toward this approach are: a) the inability to solve autonomously the lost in space problem; b) the need to run expensive and dedicated campaigns of observations to improve the knowledge of the orbital parameters of some asteroids; $c$ ) the need to have at anytime a well defined and controlled trajectory; d) the lack of robustness in isolating the asteroid.

\section{OPERATIONAL SCENARIOS}

The method devised by the authors overcomes these limitations and features the following capabilities: a) fully autonomous and on-board preliminary position determination based on a single image; b) fully autonomous and on-board accurate orbit determination based on several observations; c) full sky coverage up to $40 \mathrm{AU}$, beyond the solar system, the method degrades gracefully.

The method takes advantage of one of the $\mu$ ASC characteristic feature: the capability to track all the stars in the FOV above a certain magnitude and to point out any luminous object not being a star (12), i.e. any non-stellar objects including the planets. This list of objects, that provides the apparent right ascension and declination on 


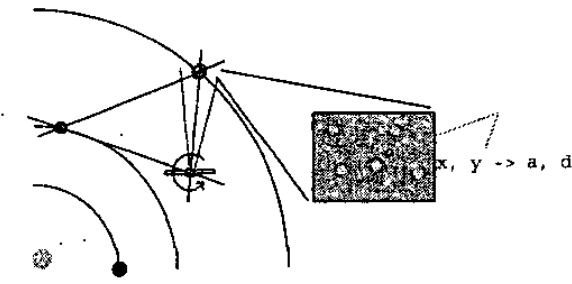

Figure 3. Ecliptic scan and detection of planets

the firmament, is matched to the very accurate planetary ephemerides stored in the $\mu \mathrm{ASC}$ to measure the spacecraft position.

Consider now a fully autonomous deep space mission. During the normal cruising phase the measurements of the position and velocity can be done periodically with a frequency that is to be defined by the required accuracy and type of orbit. Since the time and the spacecraft dynamical state are known, the on-board autonomy can choose what are the optimum planets to be observed to update the navigation. This principle is similar to the one adopted by DS- 1 but in this case the system is far mote robust and simplified because the target is chosen on-board rather than being a specific one planned on-ground before launch.

Should the need arise or after exiting the hibernation, the recovery of the dynamical state can be performed rather simply by scanning the ecliptic. Indeed, the analysis has shown that anywhere in the solar system some of the planets are always visible." Moreover, such maneuver would be quite simple as it only requires the knowledge of the inertial attitude that is measured by the $\mu \mathrm{ASC}$.

It is important to stress that such capability could be implemented and be of great interest also to any deep space spacecraft controlled from ground. Indeed, in case of permanent and unplanned loss of contact, the on-board FDIR could foresee a self orienting mode in which the spacecraft is instructed to autonomously determine its position and to point the antennae toward Earth. This would require the following steps: a) scan the ecliptic with the $\mathrm{CHU}$ of the $\mu \mathrm{ASC}$ to detect at least one planet and to measure the spacecraft position (fig 3); b) get the Earth ephemerides from the $\mu \mathrm{ASC}$; ) compute and command the rotation necessary to point the antenna to Earth

The $\mu \mathrm{ASC}$ and its DSN module offers several benefits. The system is scalable and extreme accuracy can be achieved by adopting different optics. For instance, with a focal length 12.5 times longer than the standard $\mathrm{CHU}$, ground tests have shown an accuracy of 50mas (milliarcsec) in the determination of the angular position of the planet that is an improvement of at least a factor of 20 in the measurement of the position.

The exploitation of the multi CHU philosophy of the $\mu$ ASC allows the same instrument to be used for widely - diverse purposes at the same time. E.g. 3-axes accurate attitude determination by removing the intrinsic larger error about the $\mathrm{CHU}$ boresight, contemporary observation of diverse part of the sky and objects, ultra high accuracy
Table 1. Percentage of visibility of the planets for two different limiting magnitudes:

\begin{tabular}{lrr}
\hline Planet & $\mathbf{m}_{v}=6$ & $\mathrm{~m}_{v}=7$ \\
\hline Mercury & $39.0 \%$ & $62.3 \%$ \\
Venus & $88.5 \%$ & $92.3 \%$ \\
Earth & $80.5 \%$ & $87.6 \%$ \\
Mars & $24.0 \%$ & $38.2 \%$ \\
Jupiter & $94.7 \%$ & $96.1 \%$ \\
Satum & $93.2 \%$ & $95.4 \%$ \\
Uranus & $27.9 \%$ & $55.3 \%$ \\
Neptune & $3.9 \%$ & $10.7 \%$ \\
Pluto & $0 \%$ & $0.03 \%$ \\
\hline
\end{tabular}

attitude and/or position measurements - by using different optics - or may be used for high accuracy trajectory determination of other detected objects, such as e.g. asteroids.

Another beauty of this method is that the CHUs do not need to be inter-calibrated to be pointed accurately to a specific target or to reconstruct the measurements because the information is directly available and obtainable in the inertial frame.

Other applications of the multi CHU $\mu \mathrm{ASC}$ useful to "Bering" and deep space missions are described in (8), (9) \& (4).

\section{PRELIMINARY ANALYSES AND GROUND TESTS RESULTS}

The key elements of the DSN module have been designed, implemented in the $\mu \mathrm{ASC}$ and tested.

The main goals of the tests were to assess: a) the robustness of the method to solve the lost-in-space problem; b) the preliminary accuracy achievable with a single planet and single observation; c) the visibility of the planets throughout the solar system and d) that the implementation of the DSN module into a $\mu$ ASC does not degrade the performance and attitude measurements.

The visibility was verified by computing the planet magnitude $\mathrm{m}_{v}$ for different phase angles and distances. The results are given in table 1 .

The two major planets are not visible when the spacecraft is quite far from the Sun, well beyond Uranus and almost in lower conjunction, i.e. Sun-planet-spacecraft are almost aligned. Jupiter, Satum and the Sun are aligned approximately every $2 \mathrm{l}$ years. So frequent is the risk of not having both of them not visible.

In order to have a preliminiry assessment of the performance of the DSN function, several observations of different planets were carried out at various times and sites. The first measurements were done observing Mars and the results were quite positive as a Noise Equivalent Position (NEP) of approx. $3000 \mathrm{Km}$ was measured (see fig.4). 

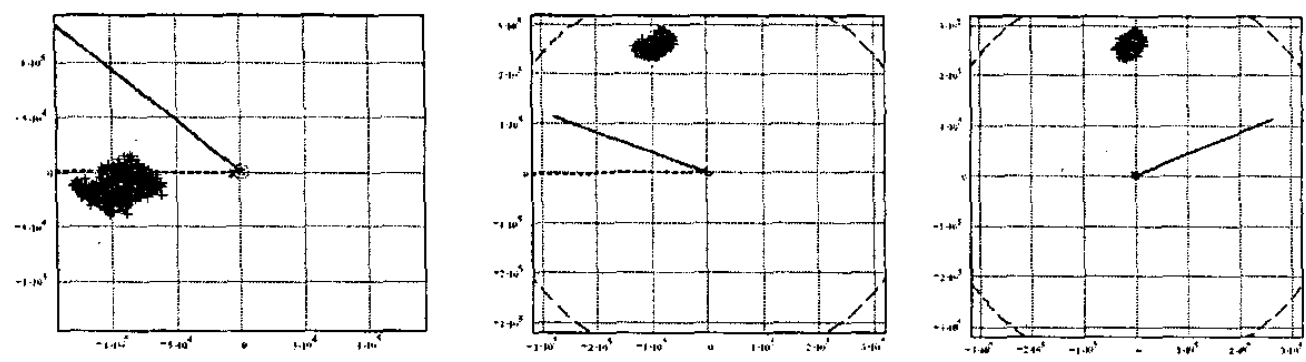

Figure 5. Single image position accuracy observing Venus. The blue points are the measured values with respect to the Earth (centre of the figure), the red line is the sun direction and the dash line is the direction to Venus. The dash circle is the orbit of the Moon. The three plots are the projections of the measurements over the three planes $x y, x z, y z$, respectively from left to right.

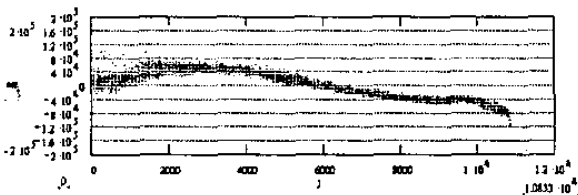

Figure 4. Noise Equivalent Position (NEP) of the measurements obtained observing Mars.

At the beginning of August 2002, at the Mauna Kea Observatories (HI-USA) two planets were useful, i.e. sufficiently above the horizon to allow for a reasonable atmospheric refraction assessment: Venus and Uranus. Venus was almost on quadrature thus offering a good geometry but low over the horizon and rapidly setting after sunset. Therefore, the images were affected by heavy absorption and moderate refraction. Both effects were not fully correctable. On the contrary, Uranus was very high over the horizon when passing the meridian. However, it was almost on the earth-sun line thus providing a poor geometry. The results of the Venus observations are shown in figure 5 . Venus is always correctly identified and the position of the observer location (Hawaii) computed. The results clearly show an offset, bias, of $115,000 \mathrm{Km}$ (mainly in the xy-plane) whereas the $3 \sigma$ dispersion of the measurements is only $18,000 \mathrm{Km}$. The dispersion of the measurements is coherent with the expectation (single image, single determination, no filter, no corrections). Whereas the bias is considerably larger than expected. There are several possible causes for it and a definitive answer has not yet been found. Also Uranus is always correctly identified and the position of the earth computed. However, the results are quite different than the ones obtained observing Venus. The errors are much bigger and there is not a well defined bias. As expected, the errors are pretty much aligned along the line of sight. However, there is also a clear time effect that seems to be related more to the observations (raw data) rather than to the computation of the position. The cause of this behavior is not yet completely understood and the analysis is still in progress.

A few images of Satum were taken in February 2003 from the observing facility at Ørsted.DTU (DK). Saturn, at the time, offered a good geometry and was high above the horizon, thus minimizing the disturbance from the atmosphere. The results of the observations are shown in fig. 6. Like Venus and Uranus, Satum is always correctly identified. The results show a bias of $485,000 \mathrm{Km}$, mainly in the direction towards the Sun). The $1 \sigma$ dispersion of the measurements is approximately $117,000 \mathrm{Km}$. These findings differ somewhat from the other two cases. The relatively larger dispersion could be explained by the small number of measurements. However, the bias is also significantly large in spite of the better geometry. Like for Venus, there are several possible causes for this and a definitive answer has not yet been identified.

\section{CONCLUSIONS \& FUTURE WORK}

Despite the observed bias terms, the preliminary results are very positive and encouraging:

- The algorithms are simple, robust and precise;

- The planet was always correctly identified when in the field of view (either Venus, Uranus or Saturn) and there was never a false lock in more than 4200 independent lost in space solutions;

- The position of the observer was always correctly computed even though the results show either a bias (Venus) and a large error ellipse (Uranus);

- At $2 \mathrm{~Hz}$, the DSN module ran smoothly without affecting the performance of the $\mu$ ASC in measuring the attitude. The only perceivable effect was the drop-out of one attitude package every ten minutes in concomitance with the update of the accurate ephemerides of all the planets;

- Areas of improvements have been identified to increase the accuracy, to improve the robustness and to reduce or eliminate the drop-out.

\section{REFERENCES}

[1] A. Andersen et al., Why Bering? Asteroidal Diversity, Origin and Transportation, Proceedings of the RAST 2003 Conference 

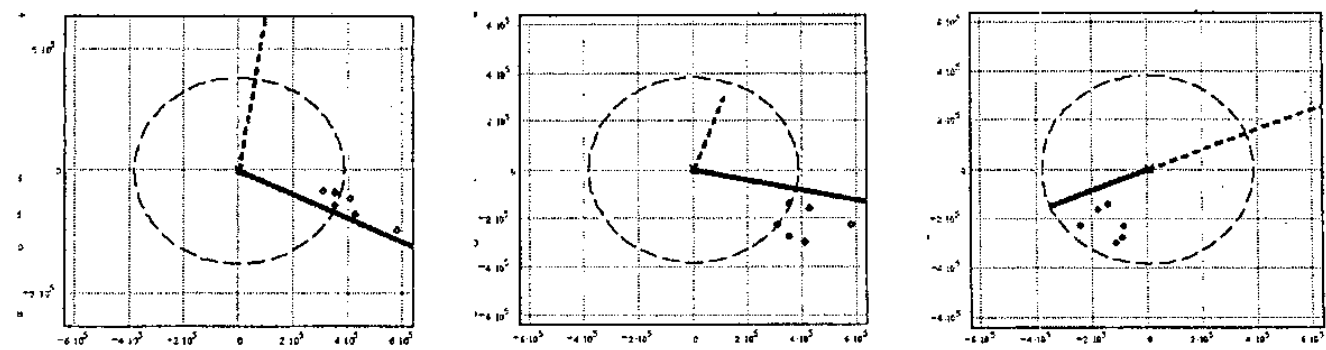

Figure 6. Single image position accuracy observing Saturn. The blue points are the measured values with respect to the Earth (centre of the figure), the red line is the sun direction and the dash line is the direction to Saturn. The dash circle is the orbit of the Moon. The three plots are the projections of the measurements over the three planes $x y, x z, y z$, respectively from left to right.

[2] A. Andersen et al., The Autonomous Asteroid Mapping Mission Bering, Proceedings of the 5th IAA International Conference on Low-Cost Planetary Missions, ESTEC 2003

[3] S. Bhaskaran et al., Orbit determination performance evaluation of the Deep Space 1 autonomous navigation system, AAS 98-193, 1295-1314

[4] T. Denver et al., The Bering Target Tracking Instrumentation, Proceedings of the RAST 2003 Conference

[5] F. Hansen, P.L. Thomsen, The Bering Mission Trade-off and Scaling, Proceedings of the RAST 2003 Conference

[6] J.L. Jørgensen et al., Enhanced Mission Performance from Autonomous Instrument Guiding, Proceedings of the '5th IAA International Conference on LowCost Planetary Missions, ESTEC 2003

[7] J.L. Jørgensen et al., MicroASC a Miniature Star Tracker, 4th International Symposium of the IAA on Small Satellites for Earth Observations, Berlin 2003

[8] J.L. Jørgensen et al., The Bering Autonomous Target Detection, Proceedings of the RAST 2003 Conference

[9] P.S. Jørgensen et al., Autonomous Target Ranging Techniques, Proceedings of the RAST 2003 Conference

[10] R. Michelsen et al., Asteroids and NEO Detection Models, Proceedings of the RAST 2003 Conference

[11] R. Michelsen et al., The Bering Small Vehicle Asteroid Mission Concept, in press in Annals of the New York Academy of Sciences.

[12] T. Riis, J.L. Jørgensen, M. Betto, Operations of a non-stellar object tracker in space, IEEE AeroSpace Conference, Denver - USA, March 1999

[13] L.G. Taff, On initial orbit determination, Astron. J. 89(9), 1426(1984)

[14] P.L. Thomsen, F. Hansen, The Bering Mission Space Segment, Proceedings of the RAST 2003 Conference 